\title{
Estudio magnético integral de flujos de lava del volcán Xitle: implicaciones arqueológicas sobre el abandono de Cuicuilco
}

\author{
Miguel Cervantes-Solano, Gerardo Gifuentes-Nava, Gecilia I. Caballero-Miranda, \\ Avto Goguitchaichvili, Héctor López-Loera, Hugo Delgado-Granados, \\ Juan Morales-Contreras, Jaime Urrutia-Fucugauchi
}

\begin{abstract}
Miguel Cervantes-Solano
miguel_cervantes@enesmorelia.unam.mx

Escuela Nacional de Estudios Superiores Unidad Morelia, Universidad Nacional Autónoma de México, Antigua Carretera a Pátzcuaro 8701, Ex-Hacienda de San José de La Huerta, 58190 Morelia, Michoacán, México.

\section{Gerardo Gifuentes-Nava \\ Cecilia I. Caballero-Miranda \\ Avto Goguitchaichvili \\ Juan Morales-Contreras}

Laboratorio Interinstitucional de Magnetismo Natural, Instituto de Geofísica Unidad Michoacán, Universidad Nacional Autónoma de México, Antigua Carretera a Pátzcuaro 8701, Ex-Hacienda de San José de La Huerta, 58190 Morelia, Michoacán, México.
\end{abstract}

\section{Héctor López-Loera \\ División de Geociencias Aplicadas, IPICYT, Camino a La Presa de San José 2055, Lomas 4 sección, 78216 San Luis, San Luis Potosí, México.}

\section{Hugo Delgado-Granados \\ Departamento de Vulcanología, Instituto de Geofísica, Universidad Nacional Autóno- ma de México, Circuito de la investigación Científica s/n, Ciudad Universitaria, 04150 Coyoacán, CDMX, México.}

\section{Jaime Urrutia-Fucugauchi}

Laboratorio de Paleomagnetismo, Instituto de Geofísica, Universidad Nacional Autónoma de México, Circuito de la investigación Científica s/n, Ciudad Universitaria, 04150 Coyoacán, CDMX, México.

BOL. SOC. GEOL. MEX. 2019

VOL. 71 NO. 2

P. $397-411$

http://dx.doi.org/10.18268/BSGM2019v71n2a10

Manuscrito recibido: Diciembre 31, 2017.

Manuscrito corregido: Mayo 20, 2018.

Manuscrito aceptado: Mayo 30, 2018.

\section{RESUMEN}

El volcán Xitle forma parte del campo volcánico Cuaternario Chichinautzin, localizado al sur del Valle de México. Sus lavas se emplazaron sobre el centro arqueológico de Cuicuilco, uno de los primeros asentamientos urbanos de México del periodo Preclásico. La erupción del Xitle tuvo un gran impacto, ya que ocasionó grandes cambios económicos y sociales favoreciendo la consolidación de Teotihuacán como la ciudad principal en el Valle de México. Diversos estudios radiométricos sitúan la edad de la erupción entre los 4000 y los 1500 años AP (Antes del Presente) y son evidencia de que Cuicuilco fue abandonado a causa de una erupción entre los años 200 BC (Before Christ / antes de nuestra era) y $200 \mathrm{AD}$ (Anno Domini / de nuestra era) sin definir con suficiente exactitud hasta ahora la edad de este evento, por lo que contribuir a la precisión de la edad de la erupción continúa siendo tema de gran interés. A continuación se presentan nuevos resultados de una datación arqueomagnética realizada a muestras de roca provenientes de una secuencia de flujos de lava del volcán Xitle, localizada al sur del Valle de México, en las inmediaciones del campus de Ciudad Universitaria (CU) de la Universidad Nacional Autónoma de México (UNAM). Para las determinaciones se usó un vector geomagnético completo y se empleó el modelo de predicción de campo SHA.DIF.14K. Los resultados de la datación permiten identificar dos periodos eruptivos, el primero entre los años 1742 y 1491 BC y el segundo relacionado con el volcán Xitle, comprendido entre 79 BC y 595 AD. La edad del segundo periodo es compatible con la posibilidad de un abandono paulatino de Cuicuilco y coincide con las edades aceptadas para el periodo eruptivo más importante del volcán.

Palabras clave: Xitle, Arqueomagnetismo, Guiculco, Valle de México.

\section{ABSTRACT}

The Xitle volcano is part of the Quaternary Chichinautzin volcanic field, located in the southern portion of the Valley of Mexico. The Xitle lavas were emplaced on the archaeological site of Cuicuilco, one of the first urban settlements of the Preclassic period in Mexico. This eruption had a great impact, since it caused great economic and social changes, favoring the consolidation of Teotihuacan as the dominant city in the Valley of Mexico. Available radiometric studies place the age of the eruption between 4000 to 1500 years BP (before present) and there is evidence that Cuicuilco abandonment was due to an eruption between $200 B C$ (Before Christ) and 200 AD (Anno Dominil after Christ) without exact definition about the age of this event. Therefore, contributing to the accuracy of the age of the eruption continues to be of great interest. The results of new archaeomagnetic dating of rock samples from a sequence of lava flows from Xitle volcano located within the campus of the University of Mexico (UNAM) campus are presented below. A full geomagnetic vector and the field prediction model SHA.DIF.14K were used for such determinations. Two eruptive periods are identified, the first between 1742 and $1491 \mathrm{BC}$ and the second, related to the eruption of Xitle volcano, between 79 BC and 595 AD. The age of the second period is compatible with the hypothesis of gradual abandonment of Cuicuilco and coincides with the accepted ages for the volcano's most important eruptive period.

Keyzords: Xitle, Archaeomagnetism, Cuicuilco, Valley of Mexico. 


\section{Introducción}

El volcán Xitle forma parte del campo volcánico Cuaternario Chichinautzin, localizado al sur del Valle de México (Herrero-Bervera et al., 1986; Urrutia-Fucugauchi y Martin del Pozzo, 1993). Es un cono monogenético cuyas lavas cubren un área aproximada de $80 \mathrm{~km}^{2}$ en el que se identifican siete unidades de flujos (Figura 1; Delgado-Granados et al., 1998). El flujo más extenso hacia el norte es de más que $13 \mathrm{~km}$ de longitud, cubre un área de 70 km² (Martin del Pozzo et al., 1997; Delgado-Granados et al., 1998) y se encuentra emplazado sobre el centro arqueológico de Cuicuilco, considerado como uno de los primeros asentamientos urbanos del Valle de México (Heizer y Bennyhoff, 1958). Desde un punto de vista arqueológico, la erupción del Xitle tuvo repercusiones muy significativas, ya que se estimaba que para el Preclásico Tardío la población que habitaba esta zona era superior a los 20000 habitantes, sin embargo, en condiciones aún no determinadas, el sitio fue abandonado y destruido a causa de una erupción entre los años 200 BC y 200 AD (Blanton et al., 1981), sin conocerse con suficiente precisión hasta ahora la edad de este evento. Existen algunas evidencias obtenidas de la exploración del basamento principal de la pirámide de Cuicuilco que sugieren que el abandono fue paulatino (Piña-Chan, 1967; Muller, 1990; Navarrete, 1991), aunque la gran mayoría del asentamiento y sus alrededores fueron cubiertos por las lavas. Los efectos de este acontecimiento ocasionaron grandes cambios en el contexto económico y social, favoreciendo la consolidación de Teotihuacán como la ciudad principal en el Valle de México (Heizer y Bennyhoff, 1958). Por este motivo, conocer con precisión la edad de la erupción del Xitle continúa siendo de gran interés.
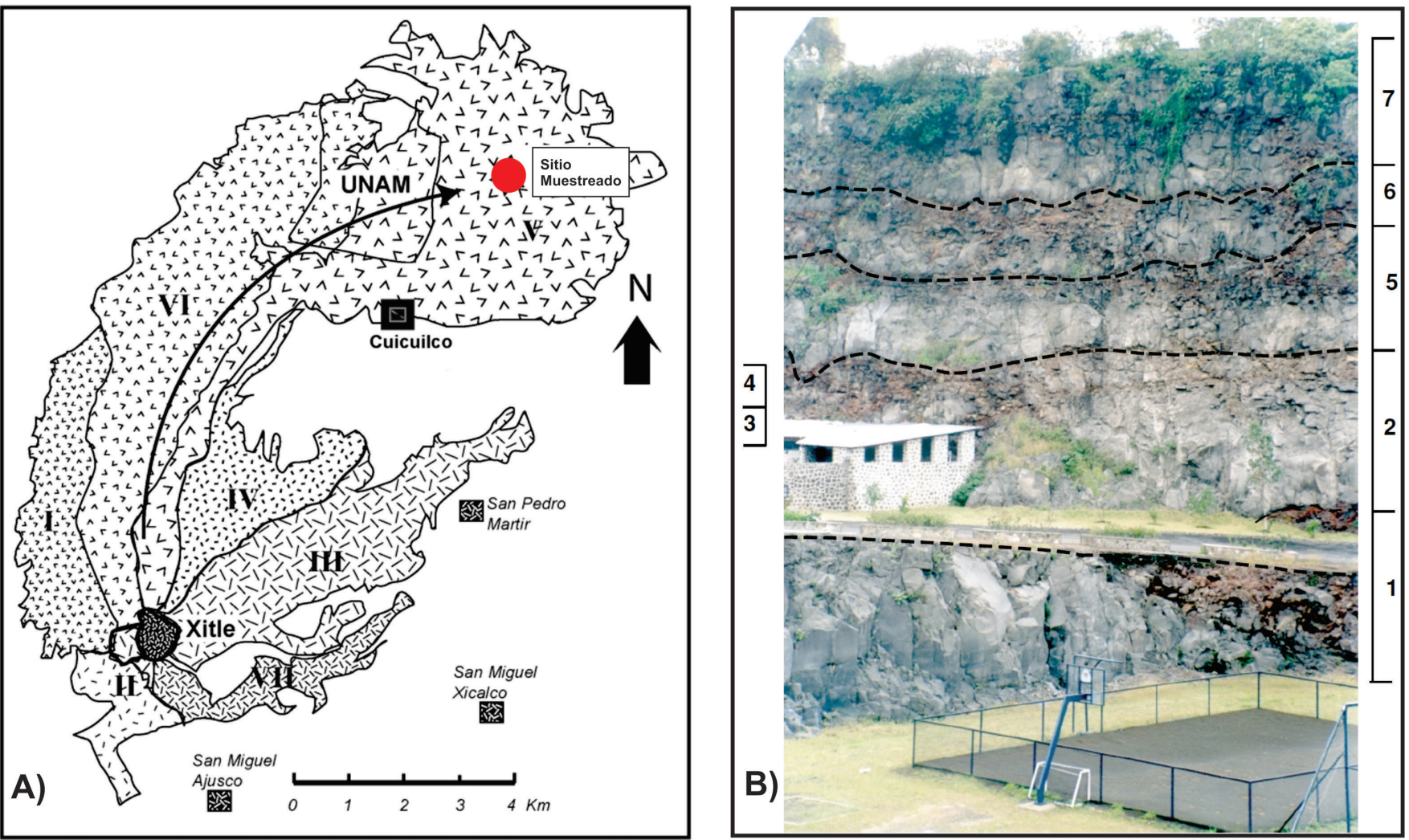

Figura 1 A) Mapa esquemático del volcán Xitle; la flecha indica la dirección inferida del flujo, los números romanos las unidades de lava identificadas en orden estratigráfico (Delgado-Granados et al., 1998) y el círculo de color rojo indica el sitio de muestreo el cual corresponde a la unidad V; B) Identificación de los flujos estudiados y su relación estratigráfica (modificado de Alva-Valdivia, 2005). 
Actualmente las edades obtenidas mediante radiocarbón se agrupan alrededor de los 2000 años BP (Delgado-Granados et al., 1998; González et al., 2000; Siebe, 2000) pero existen determinaciones individuales con edades desde los 4000 hasta 1500 años BP (Urrutia-Fucugauchi, 1996). Más recientemente, Urrutia-Fucugauchi et al. (2016) reportaron una edad obtenida por el método de datación arqueomagnética de $2086 \mathrm{BP}$ con un intervalo de 95\% de confianza entre 1995 y 2177 años $\mathrm{BP}$, edad que refuerza la hipótesis de la relación entre el abandono de Cuicuilco y la erupción del Xitle. Hasta ahora este volcán ha sido considerado como monogenético y se desconoce la duración de la erupción, aunque se supone que puede ser del orden de una década, similar a la duración de las erupciones históricas del volcán Paricutín, de 1943 a 1953 (Luhr y Simkin, 1993) y el volcán Jorullo, de 1759 a 1774 (Bullard, 1976; Luhr y Carmichael, 1985). Alva-Valdivia (2005) reporta una edad $\mathrm{C}^{14}$ de 1960-65 años BP a partir de una muestra de paleosuelo obtenida en la base de esta sección, mientras que Siebe (2000) reporta una edad también de radiocarbón de $1670 \pm 35$ años $\mathrm{BP}$ para la erupción. Estas determinaciones y la dispersión en las más de 30 edades reportadas (Urrutia-Fucugauchi, 1996, 2016; Siebe, 2000) han sugerido que la duración de la erupción podría ser mayor. El objetivo de este estudio es determinar, mediante el método de datación arqueomagnética, las edades de seis flujos de lava del volcán Xitle que permitan conocer con mayor precisión su historia eruptiva, para examinar sus posibles repercusiones vulcanológicas y arqueológicas.

\section{Muestreo y estudio paleomagnético}

El muestreo se realizó en el sitio conocido como La Cantera $\left(19^{\circ} 18.784^{\prime} \mathrm{N}, 9^{\circ} 10.317^{\circ} \mathrm{O}\right)$ en las inmediaciones de la CU (Figura 1A). En este sitio se encuentra la exposición más completa de flujos de lava con un espesor de $\sim 40 \mathrm{~m}$ y corresponde a la unidad V descrita por Delgado-Granados et

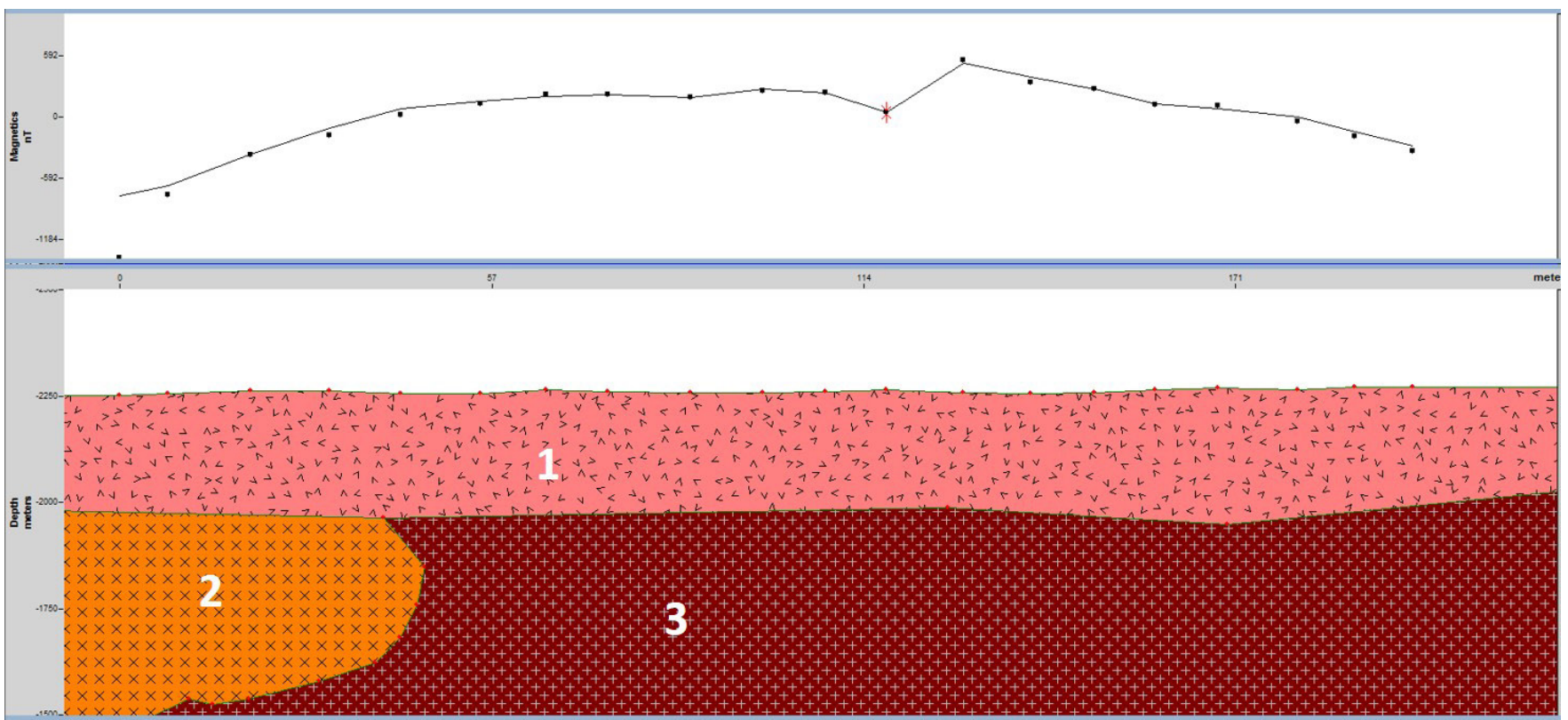

Figura 2 Modelo de la sección magnética terrestre X01 A con dirección W-E del volcán Xitle ubicada en la zona de la cantera de la UNAM; las zonas 1 y 2 representan flujos de lava 5 y 4 respectivamente, mientras que la zona 3 se utilizó para modelar el resto de los flujos en conjunto para compensar la ausencia de datos sobre la variación de las propiedades magnéticas con la profundidad. 
al. (1998), en la que se distinguen siete unidades individuales de lava (Figura 1B) con espesores de 2 a 8 m cada una. Las muestras se obtuvieron con ayuda de una perforadora portátil de gasolina; todas las muestras fueron orientadas con una brújula magnética y con la brújula solar siempre que fue posible. En total se obtuvieron 53 núcleos paleomagnéticos estándar (8 a 10 muestras por flujo), provenientes de seis flujos, y fueron numerados consecutivamente según su posición estratigráfica, siendo el Flujo 1 el que está en la parte inferior y el Flujo 6 el que está en la parte superior.

\subsection{MODELO MAGNÉTICO TERRESTRE DE LA SEGGIÓN ESTUDIADA}

Para definir la geometría y profundidad de los flujos volcánicos estudiados, se realizó un modelo 2D del campo magnético residual de la sección (identificada como X01A), el cual presenta una dirección W-E y una longitud del orden de los $200 \mathrm{~m}$. La geometría, profundidad y propiedades magnéticas de las fuentes para ajustar la sección magnética, se muestran en la Figura 2 y Tabla 1. Para realizar este modelado utilizamos el programa GM-SYSTM de Geosoft, el cual utiliza una rutina de inversión, basada en el algoritmo de Marquardt (1963), para linealizar e invertir los cálculos. GMSYSTM utiliza una aplicación de este algoritmo para datos magnéticos, proceso desarrollado por el Servicio Geológico de los Estados Unidos (USGS) en el software SAKI (Webring, 1985). La sección magnética es modelada por cuerpos poligonales con diferentes magnetizaciones (Tabla 1).

\subsection{TRATAMIENTOS MAGNÉTICOS}

Los tratamientos magnéticos fueron realizados en las instalaciones del Laboratorio Interinstitucional de Magnetismo Natural (LIMNA) del Instituto de Geofísica de la UNAM. Todas las muestras fueron cortadas en especímenes paleomagnéticos cilín-
Tabla 1. Resumen de los datos paleomagnéticos calculados por flujo. Dec. es la declinación magnética promedio, Inc. es la inclinación magnética promedio, $k$ y $\alpha_{95}$ son el parámetro de precisión y el ángulo del $95 \%$ de confianza de la estadística de Fisher.

\begin{tabular}{|l|c|c|c|c|c|}
\hline Flujo & $\mathbf{N} / \mathbf{n}$ & $\begin{array}{c}\text { Dec } \\
\left({ }^{\circ}\right)\end{array}$ & $\begin{array}{c}\text { Inc } \\
\left({ }^{\circ}\right)\end{array}$ & $\begin{array}{c}\boldsymbol{\alpha}_{95} \\
\left({ }^{\circ}\right)\end{array}$ & $\mathbf{k}$ \\
\hline Flujo 1 & 8-Aug & 355.8 & 31.8 & 2.6 & 443 \\
\hline Flujo 2 & 8-Aug & 347.4 & 33 & 3.6 & 233 \\
\hline Flujo 3 & 8-Aug & 354.5 & 37.6 & 5 & 124 \\
\hline Flujo 4 & 8-Aug & 4.1 & 34.5 & 5.2 & 116 \\
\hline Flujo 5 & 8-Aug & 5.8 & 38.7 & 4.2 & 172 \\
\hline Flujo 6 & 8-Aug & 357.9 & 32 & 7.9 & 50 \\
\hline
\end{tabular}

dricos estándar y colocadas dentro de un blindaje magnético durante dos semanas para reducir la posibilidad de una remagnetización de origen viscoso. Posteriormente se les midió la magnetización natural remanente (MNR) con un magnetómetro de giro AGICO JR5. Para examinar la estabilidad magnética y la dirección de la magnetización remanente característica (ChRM), las muestras fueron sometidas a desmagnetización progresiva mediante campos alternos de 10 a 12 pasos con una intensidad máxima de $90 \mathrm{mT}$ utilizando un desmagnetizador GSD Schonstedt. Las direcciones características (ChRM) de cada muestra se determinaron mediante el análisis de componentes principales (PCA; Kirschvink, 1980), mientras que las direcciones promedio de cada sito fueron calculadas siguiendo la estadística de Fisher (1953). En total se desmagnetizaron 48 muestras $(8$ por flujo). En la mayoría de las muestras estudiadas $(60 \%)$ se aprecian dos componentes de magnetización, la primera de ellas es removida durante los primeros pasos de desmagnetización (campos de $5 \mathrm{mT}$ a $10 \mathrm{mT}$ ) y representa menos del $10 \%$ del valor de la magnetización total de las muestras y probablemente sea de origen viscoso. Una vez removida esta componente, el comportamiento es lineal hacia el origen de coordenadas en los 

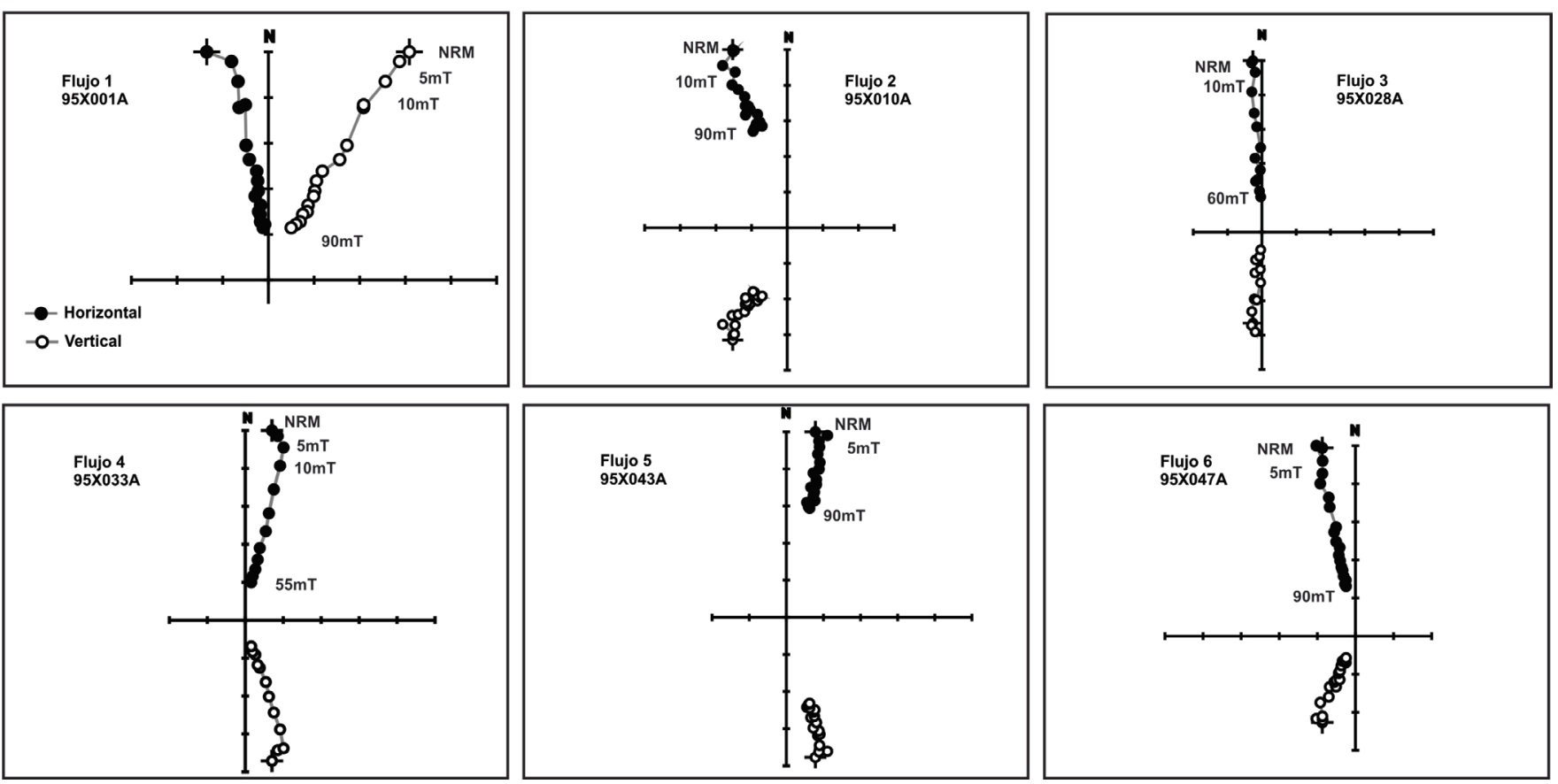

Figura 3 Diagramas ortogonales de desmagnetización representativos de los flujos de lava estudiados. Los números indican el valor máximo del campo magnético aplicado en cada paso de desmagnetización.

diagramas ortogonales de Zijderveld (1967). Otro grupo de muestras $(31 \%)$, correspondientes a los flujos 2 y 5 , conservan cerca del 40 al 50\% de la magnetización inicial luego de haberles aplicado campos magnéticos de $90 \mathrm{mT}$, sin embargo, se observa también un comportamiento lineal hacia el origen de coordenadas. En el 9\% restante de las muestras se identificó una sola componente estable con un comportamiento unidireccional hacia el origen. En todos los casos fue posible aislar las componentes direccionales de la magnetización remanente característica ChRM (6 a 12 puntos) con una máxima desviación angular $\mathrm{MAD}<4^{\circ}$, por lo que las considera de origen primario. En la Figura 3 se muestran gráficas representativas de las desmagnetizaciones. Posteriormente se obtuvieron los promedios de Fisher (1953) para cada flujo con valores de incertidumbre $a_{95}$ menores a $8^{\circ}$, lo cual indica buena precisión en las determinaciones. El resumen de estos resultados se muestra en la Tabla 2 y en la Figura 4.

\subsection{MAGNETISMO DE ROGAS}

Para determinar la naturaleza y estabilidad térmica de los portadores magnéticos presentes en las rocas estudiadas, se llevaron a cabo experimentos de susceptibilidad magnética en función de la temperatura (curvas K-T). Se utilizó un susceptibilímetro AGICO Kappa Bridge modelo MFK1. Una muestra de cada flujo fue calentada desde la temperatura ambiente hasta $\operatorname{los} 620^{\circ} \mathrm{C}$ a un ritmo de $20^{\circ} \mathrm{C} / \mathrm{min}$. en presencia de gas argón para reducir los efectos de la oxidación durante el calentamiento y posteriormente enfriada al mismo ritmo. Las curvas termomagnéticas indican la existencia de una fase magnética durante el calentamiento con temperaturas de Curie entre los $520^{\circ} \mathrm{C}$ y $560^{\circ} \mathrm{C}$, lo cual sugiere magnetita o titanomagnetita con contenido bajo en titanio como la responsable de la magnetización. Las curvas de enfriamiento son similares a las de calentamiento mostrando una disminución de la susceptibilidad 
Tabla 2. Datos de inclinación (Inc.), declinación (Dec.), susceptibilidad magnética (K) y magnetización (Mag.) utilizados para ajustar la sección magnética terrestre X01A en el modelo de la Figura 2.

\begin{tabular}{|c|c|c|c|c|}
\hline Flujo & Inc $\left({ }^{\circ}\right)$ & Dec $\left({ }^{\circ}\right)$ & $\boldsymbol{K}(\mathbf{S I})$ & Mag (A/m) \\
\hline $1(5)$ & 38.7 & 5.8 & 0.00896 & 5 \\
\hline $2(4)$ & 34.5 & 4.1 & 0.003 & 3 \\
\hline $3(?)$ & -- & -- & 1 & -- \\
\hline
\end{tabular}

inicial menor al 15\%, la cual es probablemente producto de la oxidación producida durante el calentamiento (Figura 5).

\subsection{PALEOINTENSIDAD}

La determinación de la paleointensidad (PI) se realizó siguiendo el método de doble calentamiento de Thellier (Thellier y Thellier, 1959) modificado por Coe (1967). Solo las muestras que mostraron una sola componente direccional de NRM en los diagramas ortogonales de Zijderveld (1967) y una variación menor al $15 \%$ en los valores de la susceptibilidad magnética entre el calentamiento y enfriamiento fueron seleccionadas. En total se usaron 42 muestras para los experimentos de paleointensidad. Las muestras fueron calentadas en 12 pasos de temperatura entre los $100^{\circ}$ y $580^{\circ} \mathrm{C}$ y se aplicó un campo de laboratorio de $45 \mu \mathrm{T}$ paralelo al eje $\mathrm{Z}$ empleando un desmagnetizador ASC TD48.

Para garantizar la confiabilidad de los resultados en la determinación de la PI se utilizaron los siguientes criterios de selección para que una determinación individual fuera considerada como aceptable y ser incluida en el cálculo del promedio. (i) El número de puntos utilizados en el ajuste lineal del diagrama NRM-pTRM debe ser de al menos 5 ( $\mathrm{N} \geq 5$ ); (ii) debe utilizarse por lo menos el $40 \%$ del total de la NRM original (parámetro f, Coe et al., 1978); (iii) el factor de calidad q $>5$ (Coe et al., 1978); (iv) el radio b (error estándar/ pendiente absoluta de la línea de mejor ajuste del diagrama de Arai) < 0.1; (v) los resultados de paleointensidad obtenidos de los diagramas NRMpTRM no deben tener forma cóncava, debido a que en esos casos es probable que la remanencia esté asociada con la presencia de granos multidominio (Levi y Merrill, 1978) y (vi), el cálculo del promedio por flujo debe incluir al menos 3 determinaciones individuales con una desviación estándar d.s. $<10 \mu$ T. Los resultados de la PI promedio obtenida para cada flujo se muestran en la Tabla 3 y en la Figura 6 las gráficas representativas.

\subsection{DATAGIÓN ARQUEOMAGNÉTICA}

La datación arqueomagnética se obtuvo utilizando la herramienta "Archaeodating" (Pavón-Carrasco et al., 2011), a partir del modelo de variación del campo magnético Terrestre SHA.DIF.14k propuesto por Pavón-Carrasco et al. (2014). Este modelo permite hacer una comparación de los datos direccionales y de intensidad de los flujos estudiados con los de la curva de variación paleosecular correspondiente y proporciona un intervalo de edad estadísticamente posible y su nivel de confianza. Este método de datación ha sido utilizado con resultados favorables para obtener edades de flujos de lava de erupciones volcánicas recientes (Mahgoub et al., 2017; García-Quintana et al., 2016; Böhnel et al., 2016; Pérez-Rodríguez et al., 2015). Una característica de este método de datación es que proporciona intervalos de edades posibles dentro de los cuales es probable que la edad "real" se encuentre a un nivel del 95\% de confianza, por lo que en las determinaciones es común encontrar varios intervalos de edades con la misma probabilidad. Las gráficas de probabilidad y los intervalos de edades encontrados para los flujos estudiados se pueden ver en la Figura 7. Con la finalidad de realizar una interpretación global de las dataciones, se tomó en cuenta tanto la densidad de probabilidad mostrada en las grá- 
ficas de cada determinación individual como el contexto estratigráfico de los flujos. De esta forma se pueden descartar a algunos de los intervalos de edades probables de los flujos, es decir, el flujo en la parte inferior de la secuencia (Flujo 1) es el que fue emplazado primero y en consecuencia el intervalo aceptado de la edad arqueomagnética deberá ser el más antiguo entre todas las determinaciones; del mismo modo el flujo consecutivo adyacente (Flujo 2) se asume que es más joven que el primero, pero al mismo tiempo este flujo deberá ser más antiguo que el tercero (Flujo 3), y así respectivamente para el resto de los flujos. La secuencia propuesta es la que se indica en la Figura 8.

\section{Discusión de los resultados de la datación}

La mayoría de las edades radiométricas para la erupción del Xitle están agrupadas alrededor de los 2000 años BP (Urrutia-Fucugauchi, 1996; Siebe, 2000; Urrutia-Fucugauchi et al., 2016), por lo que es posible que el emplazamiento de los flujos de lava estudiados sucediera en un inter- valo muy estrecho de tiempo, sin embargo, existen también determinaciones radiométricas que arrojan una antigüedad cercana a los 4000 años BP (Fergusson y Libby, 1963, 1964) así como la edad más joven de 1670 BP encontrada por Siebe (2000). Para fines de la datación arqueomagnética se puede suponer que los flujos de lava estudiados son independientes y que fueron emplazadas con una diferencia de tiempo suficiente, la cual permite distinguir las características del campo magnético de la Tierra registrado en ellas, por lo que es posible asociarles una edad arqueomagnética individual a partir de los datos paleomagnéticos direccionales y de intensidad encontrados. Se determinaron las direcciones características en la totalidad de las muestras estudiadas y se obtuvo el promedio paleomagnético de cada uno de los seis flujos de lava con una certeza del 95\% $\left(a_{95}\right)$ entre $2.6^{\circ}$ y $7.9^{\circ}$. Los experimentos de paleointensidad produjeron, luego de aplicar los criterios de selección antes mencionados, 38 determinaciones individuales y se obtuvo el promedio estadístico con su desviación estándar para los seis flujos con valores de PI entre los 43.74 y $73.04 \mu \mathrm{T}$ y desviaciones estándar de entre 5.19 y $12.12 \mu \mathrm{T}$.
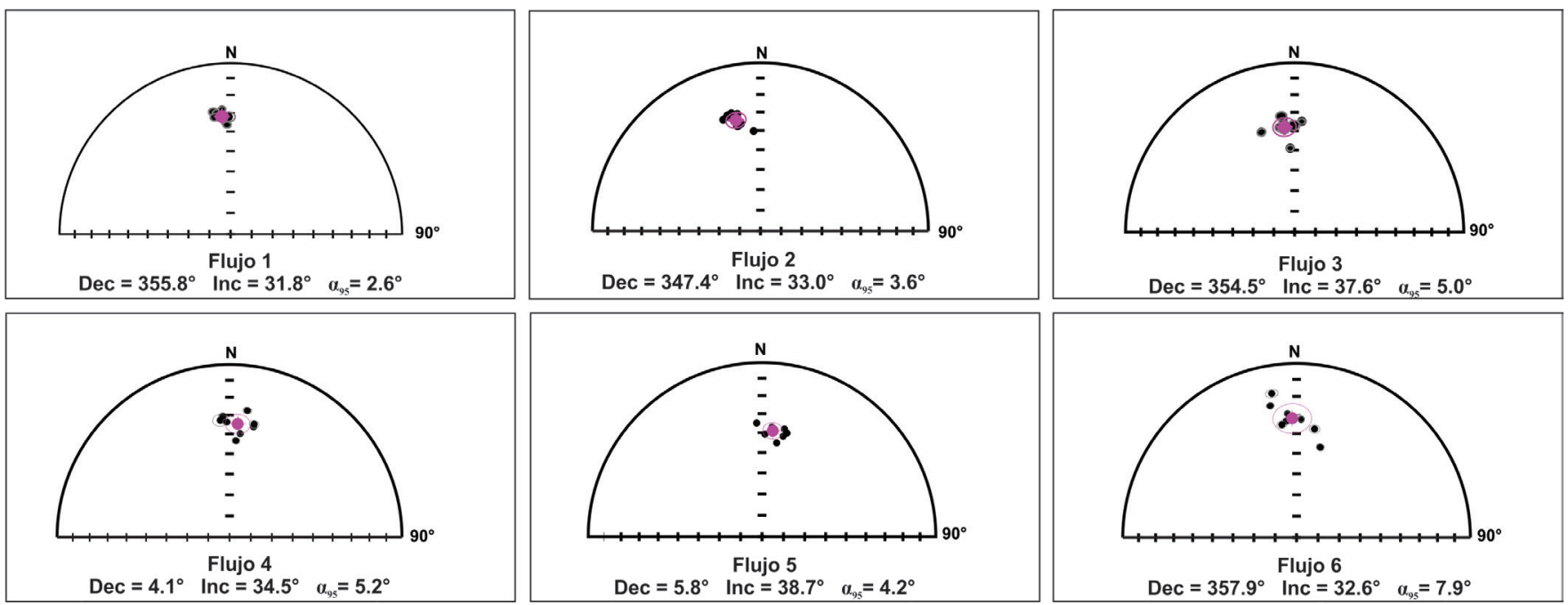

Figura 4 Diagramas ortogonales de áreas iguales con las direcciones paleomagnéticas promedio para los flujos estudiados. Dec. es la declinación magnética promedio, Inc. es la inclinación magnética promedio y $\alpha_{95}$ el ángulo del $95 \%$ de confianza de la estadística de Fisher. 

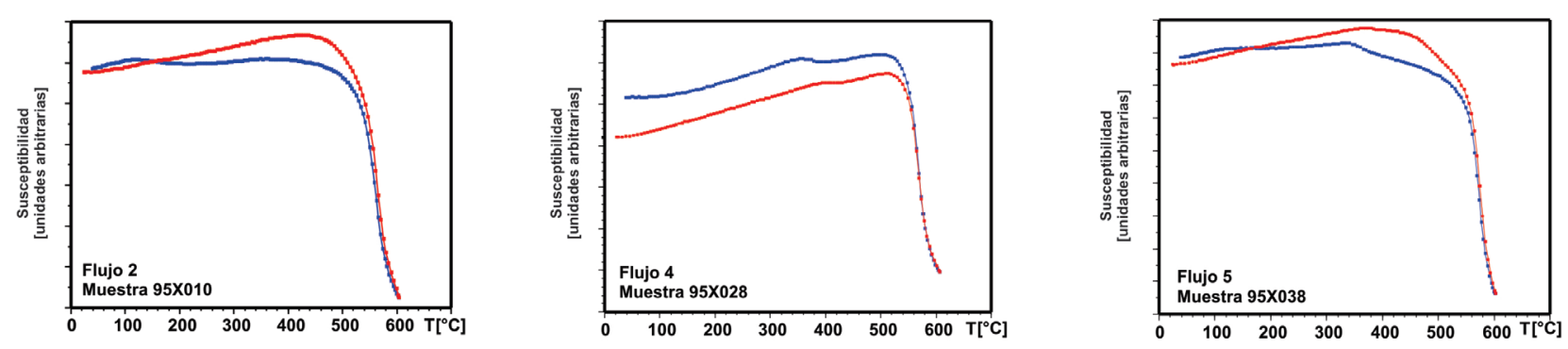

Figura 5 Curvas termomagnéticas continuas K-T. Las curvas de color rojo indican el calentamiento y las rojas el enfriamiento; la susceptibilidad magnética se muestra en unidades arbitrarias.

Luego de hacer las interpretaciones estratigráficas discutidas anteriormente, se obtiene para el Flujo 1 (Dec $=355.8^{\circ}$, Inc $=31.8^{\circ}, a_{95}=2.6^{\circ}$, PI $=43.79 \pm 8.78 \mu \mathrm{T})$ un intervalo de edad probable de [1634 - 1491] BC (3584 - 3141 BP). Esta edad es considerablemente más antigua a la edad reportada por Alva-Valdivia (2005; 1960 - 65 BP) obtenida de una muestra de carbón de la base de este mismo flujo y es más próxima al grupo de edades radiométricas más antiguas reportado por Urrutia-Fucugauchi (1996), cercano a los 4000 BP. La diferencia puede ser atribuida a que esas determinaciones se realizaron mediante el método de $\mathrm{C}^{14}$ a partir de muestras de carbón asociado a la formación rocosa o del paleosuelo que se encuentra a su alrededor, por lo que, debido al origen del carbón presente en las muestras, es posible que sean temporalmente distintas. En cambio, la datación de este estudio fue hecha utilizando muestras de roca del afloramiento, por lo cual es más probable que esta edad corresponda al inicio de la actividad volcánica eruptiva.

En el caso del Flujo $2\left(\mathrm{Dec}=347.4^{\circ}\right.$, Inc $=33.0^{\circ}$, $\left.a_{95}=3.6^{\circ}, \mathrm{PI}=63.30 \pm 12.12 \mu \mathrm{T}\right)$ el intervalo de edad más probable es de [1742 - 1626 BC] (3692 - 3576 BP). Debido al elevado valor en la incertidumbre de la PI, este intervalo de edad es más grande y se superpone al propuesto para Flujo 1. La similitud entre los datos direccionales y sus in- certidumbres sugieren que los flujos 1 y 2 pudieron haber sido emplazados en un periodo muy corto y a falta de mayor precisión en la PI se les puede considerar contemporáneos. Aun así, la combinación de ambas edades establece un límite temporal para el inicio de este periodo eruptivo entre los 1742 a 1491 años BC (3692 - 3441 BP).

Dado que estas determinaciones se hicieron en un solo sitio, sin haber hecho una comparación con flujos de lava similares en otras localidades, debido a que esta exposición es única, se puede concluir que los Flujos 1 y 2 son pertenecientes a la misma erupción, pero no necesariamente provenientes del volcán Xitle, cuya naturaleza monogenética ha sido ampliamente documentada (v.g. Martin del Pozzo, 1982; Delgado-Granados et al., 1998; Siebe, 2000). Delgado-Granados et al. (1998), a partir de datos radiométricos compilados de diferentes autores, discuten la existencia de un evento eruptivo en esta zona cuya edad se determinó en $3957 \pm 113$ años BP. Estos dos primeros flujos de lava estarían relacionados a este evento eruptivo y no necesariamente relacionados con el Xitle.

El intervalo de edad probable obtenido para el Flujo $3\left(\mathrm{Dec}=354.5^{\circ}, \mathrm{Inc}=37.6^{\circ}, \mathrm{a}_{95}=5.0^{\circ}\right.$, PI $=73.04 \pm 5.19 \mu \mathrm{T})$ es de $[96 \mathrm{BC}-18 \mathrm{AD}](2046-$ 1932 BP). Esta determinación es consistente con la edad arqueomagnética reportada por Urrutia-Fucugauchi et al. (2016) de 2177 a 1995 BP, y con la 
Tabla 3. Resultados de paleointensidad. $\mathrm{N}$ es el número de pasos de calentamiento usados para la determinación de la paleointensidad, $\mathrm{T}_{\min } \mathrm{T}_{\max }$ es el intervalo de temperatura empleado para la determinación, $F \pm \Delta F$ es el valor de la paleointensidad y su correspondiente desviación estándar, $f$ es la fracción de NRM utilizada para la determinación, g y q son los factores de calidad definidos por Coe et al. (1978). En negritas se indican las determinaciones individuales descartadas para el promedio.

\begin{tabular}{|c|c|c|c|c|c|c|c|c|c|}
\hline Flujo & Muestra & $T_{\min }\left({ }^{\circ} \mathrm{C}\right)$ & $T_{\text {máx }}\left({ }^{\circ} \mathrm{C}\right)$ & $N$ & $f$ & $g$ & $q$ & $F(\mu T)$ & $\Delta F(\mu T)$ \\
\hline \multirow[t]{7}{*}{ Flujo 1} & $94 \mathrm{X} 003 \mathrm{~B}$ & 300 & 540 & 5 & 0.93 & 0.46 & 5.56 & 28.31 & 2.17 \\
\hline & 94X004B & 300 & 540 & 6 & 0.9 & 0.66 & 14.99 & 41.47 & 1.63 \\
\hline & $94 \mathrm{X} 005 \mathrm{~B}$ & 300 & 540 & 6 & 0.74 & 0.63 & 13.98 & 54.9 & 1.84 \\
\hline & $94 \mathrm{X} 006 \mathrm{~B}$ & 300 & 540 & 5 & 0.66 & 0.52 & 5.71 & 47.08 & 2.82 \\
\hline & 94X007B & 350 & 560 & 6 & 0.82 & 0.65 & 15.42 & 45.46 & 1.56 \\
\hline & $94 X 009 A$ & 200 & 450 & 5 & 0.54 & 0.72 & 12.22 & 45.54 & 7.96 \\
\hline & Promedio & & & & & & & 43.79 & 8.78 \\
\hline \multirow[t]{8}{*}{ Flujo 2} & $94 \mathrm{X} 010 \mathrm{~B}$ & 300 & 540 & 5 & 0.65 & 0.59 & 6.71 & 66.54 & 3.81 \\
\hline & $94 \mathrm{X} 011 \mathrm{~B}$ & 300 & 540 & 5 & 0.74 & 0.42 & 7.38 & 68.85 & 2.88 \\
\hline & $94 \mathrm{X} 012 \mathrm{~B}$ & 300 & 540 & 6 & 0.69 & 0.66 & 14.7 & 48.24 & 1.51 \\
\hline & $94 X 013 B$ & 300 & 500 & 6 & 0.52 & 0.69 & 5.3 & 50.16 & 3.44 \\
\hline & 94X014B & 300 & 540 & 7 & 0.64 & 0.65 & 9.18 & 59.42 & 2.69 \\
\hline & $94 \mathrm{X} 018 \mathrm{~A}$ & 300 & 540 & 7 & 0.68 & 0.74 & 29.86 & 83.75 & 1.42 \\
\hline & $94 X 019 A$ & 200 & 540 & 7 & 0.69 & 0.51 & 6.38 & 66.12 & 3.61 \\
\hline & Promedio & & & & & & & 63.3 & 12.12 \\
\hline \multirow[t]{7}{*}{ Flujo 3} & $94 \mathrm{X} 020 \mathrm{~B}$ & 300 & 560 & 6 & 0.48 & 0.68 & 9.84 & 69.31 & 2.28 \\
\hline & $94 \mathrm{X} 021 \mathrm{~B}$ & 300 & 560 & 9 & 0.58 & 0.83 & 8.47 & 75.14 & 4.28 \\
\hline & $94 \mathrm{X} 022 \mathrm{~B}$ & 200 & 560 & 9 & 0.84 & 0.84 & 16.82 & 78.43 & 3.31 \\
\hline & $94 \mathrm{X} 023 \mathrm{~B}$ & 200 & 560 & 9 & 0.63 & 0.78 & 9.46 & 75.39 & 3.89 \\
\hline & $94 \mathrm{X} 025 \mathrm{~B}$ & 300 & 560 & 7 & 0.8 & 0.75 & 12.82 & 64.36 & 3.04 \\
\hline & $94 \mathrm{X} 026 \mathrm{~B}$ & 300 & 560 & 6 & 0.89 & 3.05 & 9.78 & 75.59 & 3.05 \\
\hline & Promedio & & & & & & & 73.04 & 5.19 \\
\hline \multirow[t]{8}{*}{ Flujo 4} & $94 \mathrm{X} 030 \mathrm{~B}$ & 200 & 540 & 9 & 0.97 & 0.81 & 22.78 & 61.05 & 2.12 \\
\hline & $94 \mathrm{X} 031 \mathrm{~B}$ & 200 & 540 & 7 & 0.93 & 0.74 & 20.84 & 63.72 & 2.08 \\
\hline & $94 \mathrm{X} 032 \mathrm{~B}$ & 300 & 540 & 7 & 0.87 & 0.74 & 12.59 & 82.59 & 4.18 \\
\hline & 94X033B & 300 & 540 & 8 & 0.79 & 0.77 & 24.08 & 68.65 & 1.74 \\
\hline & 94X034B & 200 & 560 & 9 & 0.71 & 0.8 & 14.34 & 61.9 & 2.46 \\
\hline & $94 \mathrm{X} 035 \mathrm{~B}$ & 200 & 560 & 9 & 0.55 & 0.8 & 10.54 & 69.33 & 2.88 \\
\hline & $94 \times 036 \mathrm{~A}$ & 300 & 560 & 8 & 0.61 & 0.8 & 11.87 & 64.23 & 2.61 \\
\hline & Promedio & & & & & & & 67.35 & 7.42 \\
\hline \multirow[t]{8}{*}{ Flujo 5} & 94X037B & 100 & 540 & 9 & 0.53 & 0.81 & 11.42 & 76.99 & 2.87 \\
\hline & $94 \mathrm{X} 038 \mathrm{~B}$ & 300 & 560 & 8 & 0.47 & 0.82 & 7.66 & 62.31 & 3.16 \\
\hline & 94X039B & 300 & 540 & 6 & 0.74 & 0.39 & 5.62 & 50.87 & 2.63 \\
\hline & $94 \times 040 B$ & 200 & 540 & 8 & 0.75 & 0.69 & 10.16 & 59.51 & 3.04 \\
\hline & $94 \mathrm{X} 041 \mathrm{~B}$ & 300 & 540 & 7 & 0.74 & 0.72 & 12.09 & 65.65 & 2.87 \\
\hline & $94 \times 042 B$ & 200 & 540 & 8 & 0.76 & 0.65 & 11.86 & 56.96 & 2.39 \\
\hline & $94 \times 045 B$ & 300 & 540 & 7 & 0.73 & 0.69 & 8.46 & 57.11 & 3.38 \\
\hline & Promedio & & & & & & & 61.34 & 8.31 \\
\hline \multirow[t]{8}{*}{ Flujo 6} & 94X046B & 300 & 560 & 9 & 0.39 & 0.8 & 5.7 & 70.14 & 3.9 \\
\hline & 94X047B & 200 & 560 & 10 & 0.61 & 0.83 & 8.95 & 55.38 & 3.17 \\
\hline & 94X048B & 400 & 560 & 7 & 0.36 & 0.74 & 4.01 & 67.82 & 4.53 \\
\hline & 94X049B & 300 & 560 & 7 & 0.67 & 0.73 & 11.25 & 53.4 & 2.34 \\
\hline & $94 \mathrm{X} 052 \mathrm{~B}$ & 200 & 560 & 9 & 0.83 & 0.82 & 12.77 & 69.92 & 3.74 \\
\hline & $94 \mathrm{X} 053 \mathrm{~B}$ & 300 & 560 & 7 & 0.9 & 0.79 & 6.38 & 53.91 & 5.99 \\
\hline & $94 \mathrm{X} 054 \mathrm{~A}$ & 300 & 540 & 7 & 0.77 & 0.79 & 4.53 & 50.45 & 6.77 \\
\hline & Promedio & & & & & & & 56.61 & 7.65 \\
\hline
\end{tabular}


edad radiométrica reportada por Delgado-Granados et al. (1998) de $1977 \pm 43$ años BP y se aleja de las edades más jóvenes propuestas para la erupción del Xitle, de alrededor de los 1670 años BP (Siebe et al., 2000; González et al., 2000). Observando las edades encontradas para los flujos anteriores se aprecia que transcurrieron cerca de 1400 años entre ellas, lo que sugiere que esta secuencia de lavas representa dos periodos eruptivos, uno reciente relacionado con el Xitle y otro más antiguo, cuya fuente de origen es desconocida. La edad determinada, además, coincide con la edad propuesta para el abandono de Cuicuilco.

Para el Flujo $4\left(\mathrm{Dec}=4.1^{\circ}\right.$, Inc $=34.5^{\circ}$, a95 $=$ $5.2^{\circ}, \mathrm{PI}=67.35 \pm 7.42 \mu \mathrm{T}$ ), según el gráfico de probabilidad, existen tres intervalos de edad pro-

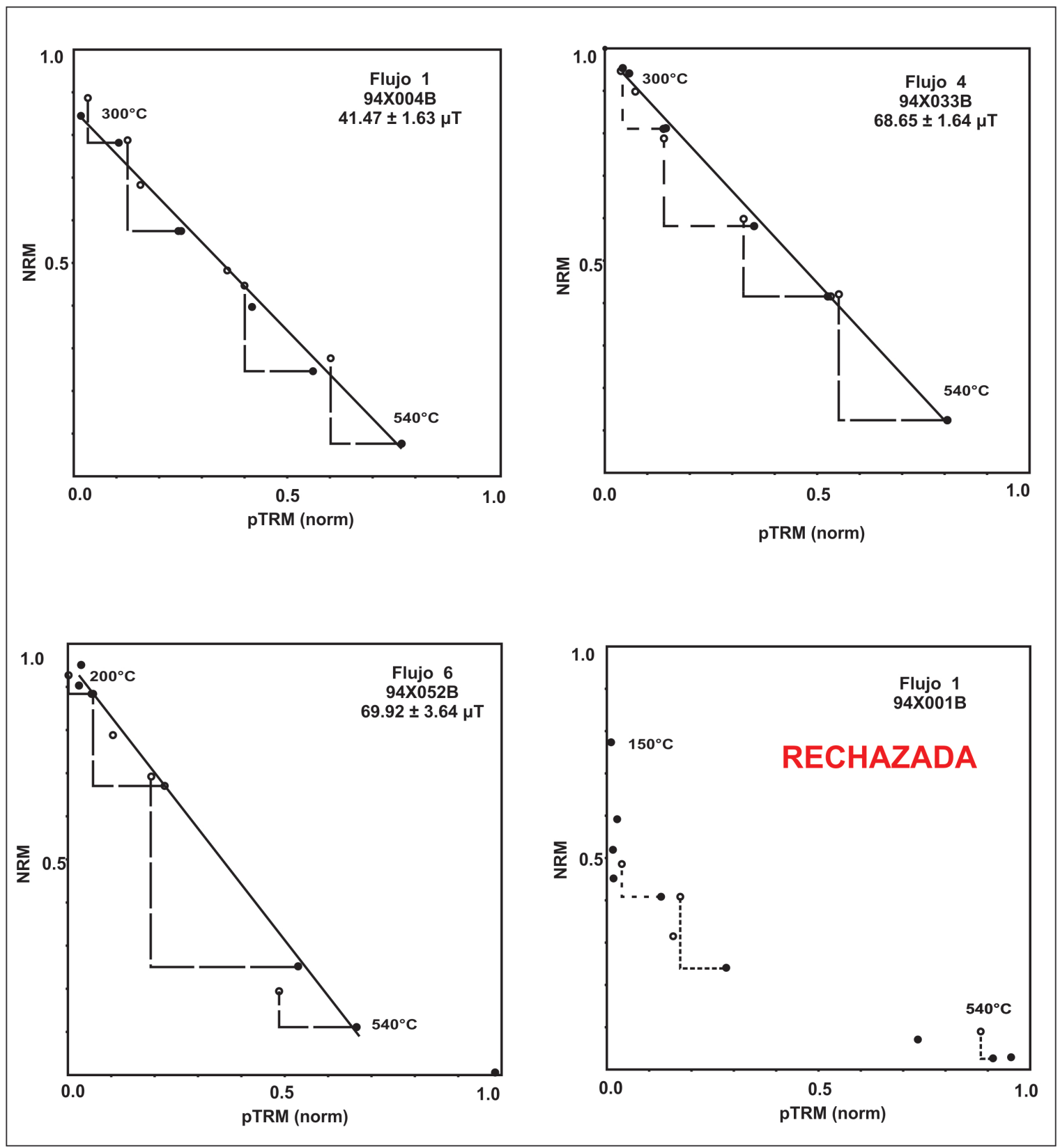

Figura 6 Diagramas de Arai de los experimentos de paleointensidad según el método de Thellier y Thellier (1959) (resultados representativos). 


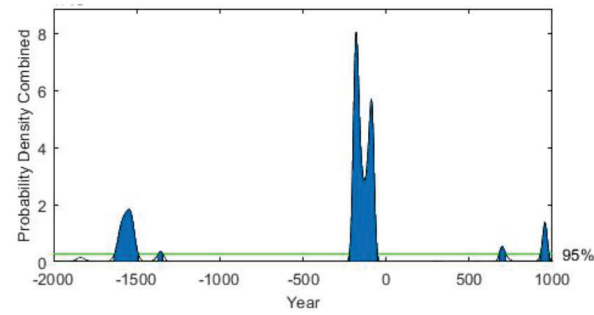

DATING: FLUJO 1

[1634BC 1491BC] [1370BC 1344BC]

[222BC 52BC]

[679AD 720AD]

[927AD 980AD]

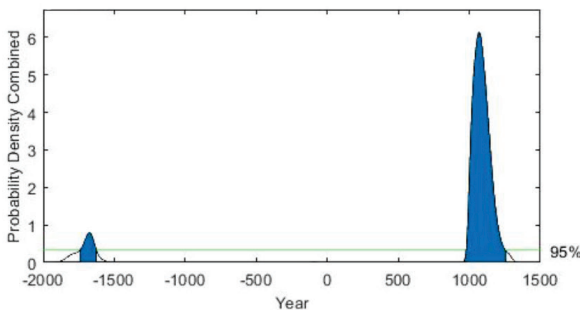

DATING: FLUJO 2

[1742BC 1626BC] [977AD 1254AD]

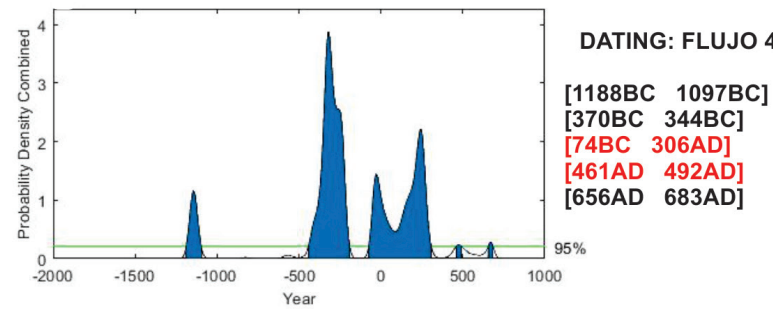

Figura 7 Datación de los flujos de lava. Se muestran las gráficas de probabilidad combinada al $95 \%$ de confianza; dentro de los corchetes se indica el intervalo de edad más probable y de color rojo el intervalo estratigráficamente compatible (véase el apartado "Discusión de los resultados de la datación"). Estas gráficas fueron hechas con la herramienta de Matlab ARCHAEODATING de Pavón Carrasco et al. (2011).

bables, dos de ellos congruentes con el contexto estratigráfico $[74 \mathrm{BC}-306 \mathrm{AD}]$ y $[461-492 \mathrm{AD}]$. El primero de ellos incluye por completo al intervalo de edad del flujo anterior, mientras que el segundo es más estrecho y supone una edad menor al flujo superior adyacente, por lo que se propone al primero como la edad probable del emplazamiento. La superposición entre las edades de los Flujos 3 y 4 sugiere que ambos se formaron duran- te un periodo muy corto comprendido entre [2024 - 1644 años BP]. Este intervalo incluye también a las edades reportadas previamente para la erupción del Xitle.

Para el Flujo $5\left(\operatorname{Dec}=5.8^{\circ}, \operatorname{Inc}=38.7^{\circ}, a_{95}=4.2^{\circ}\right.$, $\mathrm{PI}=61.34 \pm 8.31 \mu \mathrm{T}$ ) la datación y el contexto estratigráfico permiten establecer un intervalo de edad probable de [24 BC - 282 AD] el cual está completamente incluido dentro del intervalo 
de edad propuesta para el Flujo 4. Revisando los datos direccionales y las incertidumbres de estos dos flujos, se observa que tienen una distancia angular de $4.5^{\circ}$ por lo que se les considera estadísticamente idénticas y se presume que estos dos flujos se formaron casi simultáneamente.

Para el Flujo $6\left(\mathrm{Dec}=357.9^{\circ}, \mathrm{Inc}=32.0^{\circ}, \mathrm{a}_{95}=\right.$ $\left.7.9^{\circ}, \mathrm{PI}=56.61 \pm 7.65 \mu \mathrm{T}\right)$ se proponen tres intervalos de edades probables [107 - $595 \mathrm{AD}],[629$ - $769 \mathrm{AD}]$ y [799 - $1000 \mathrm{AD}]$. A falta de mayor información sobre la edad de este flujo y el siguiente, los tres intervalos son estadísticamente posibles, aunque solo el primero de ellos es compatible con el promedio de las determinaciones radiométricas para la erupción y con la edad arqueomagnética obtenida por Urrutia-Fucugauchi et al. (2016). También existe la posibilidad de que el Flujo 6 se haya formado posteriormente, por lo que puede establecerse al intervalo de 799 AD a 1000 AD como el límite en la edad del emplazamiento. El rango de edad del Flujo 6 incluye la edad reportada por Siebe (2000) de $1670 \pm 35$ años BP.

Los rangos de edad aquí reportados (Flujos 3-5) son concordantes con la edad reportada por Delgado-Granados et al. (1998), quienes determinaron la edad de la erupción del Xitle en $1977 \pm 43$ años BP.

\section{Conclusiones}

Las direcciones de magnetización característica ChRM, encontradas mediante el proceso desmagnetización por campos alternos, consideradas de origen primario, permitieron determinar el promedio direccional paleomagnético de cada uno de los flujos de lava estudiados. Con los 38 resultados individuales de los experimentos de PI, se estableció el promedio de la intensidad magnética registrada en los seis flujos. Los experimentos de

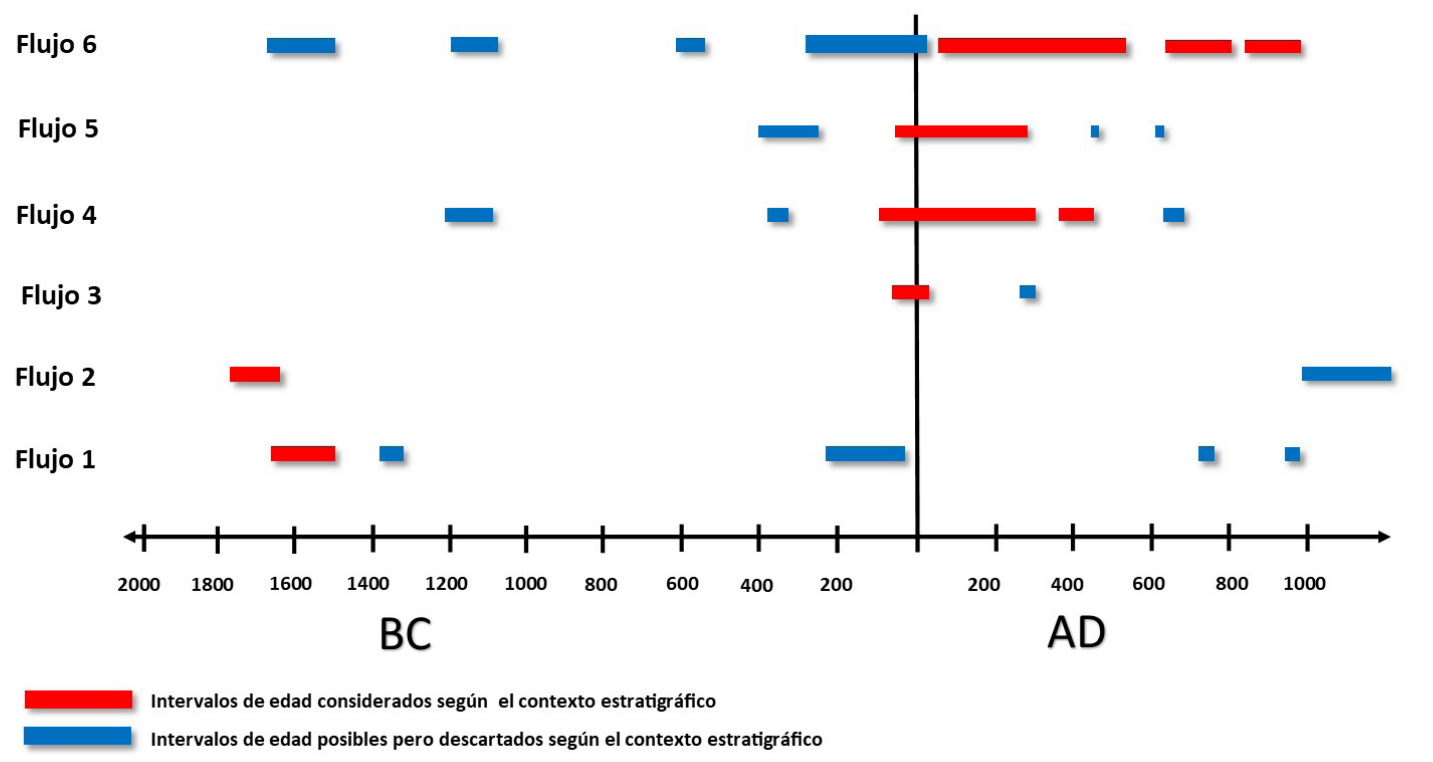

Figura 8 Secuencia de edades obtenidas por la datación arqueomagnética. Los rectángulos de color rojo indican los intervalos que concuerdan mejor con la estratigrafía, los rectángulos de color azul representan los intervalos de edad descartados. 
susceptibilidad magnética en función de la temperatura indican que la magnetita o titanomagnetita con bajo contenido en titanio es el mineral responsable de la magnetización en las rocas estudiadas. La secuencia de edades arqueomagnética presentada responde a la hipótesis de que los flujos de lava se emplazaron en tiempos distintos y representa un primer intento de asociarles edades individuales utilizando la datación arqueomagnética. Ésta, a su vez, es una herramienta basada en probabilidades, por lo que no se descarta la existencia de otras posibilidades además de que estos resultados habrán de confrontarse con las evidencias tanto vulcanológicas como arqueológicas. Las direcciones medias y las determinaciones de paleointensidad reportadas para las lavas del Xitle presentan dispersiones y rangos amplios de variación (Urrutia-Fucugauchi, 1996; Alva-Valdivia, 2005; Urrutia-Fucugauchi et al., 2016) mayores a los esperados para rocas volcánicas recientes y dado que la precisión en las dataciones depende de los registros paleomagnéticos y de la curva geomagnética de referencia (Speranza et al., 2006), estos rangos de variación inciden directamente en las dataciones magnéticas, lo que podría explicar la amplitud de los rangos de las edades aquí encontradas.

En términos generales y a juzgar por las edades previamente discutidas se identifican dos periodos eruptivos, el primero de ellos entre los 1742 a 1491 años BC y comprende a los Flujos 1 y 2 y el segundo periodo, asociado al volcán Xitle, comprendido entre $79 \mathrm{BC}$ y $595 \mathrm{AD}$, durante el cual se formaron los Flujos del 3 al 6. Esta propuesta es compatible con la evidencia sobre el abandono de Cuicuilco y su relación con un periodo eruptivo más significativo del volcán Xitle y es compatible también con la posibilidad de que el abandono haya sido paulatino ya que, si bien la erupciones fueron casi simultáneas, a partir de los intervalos de edad aquí encontrados, existe la posibilidad de que ocurrieran con la diferencia de tiempo suficiente para permitir a la población regresar a la ciudad durante estos periodos.

\section{Agradecimientos}

Este trabajo fue apoyado por el proyecto UNAM PAPIIT IA106517. AG agradece el apoyo del Proyecto CONACYT No. 251249 y el apoyo parcial de UNAM PAPIIT 101717. Agradecemos también el apoyo del M. en C. David E. Torres Gaytán para la realización del modelo magnético.

\section{Referencias}

Alva-Valdivia, L., 2005, Comprehensive paleomagnetic study of a succession of Holocene olivine-basalt flow: Xitle Volcano (Mexico) revisited: Earth, Planets and Space, 57, 839-853.

Blanton, R.E., Kowalewski, S.A., Feinman, G., Appel, J., 1981, Ancient Mesoamerica: Cambridge, Cambridge University Press, $300 \mathrm{p}$.

Böhnel, H., Pavón-Carrasco, F.J., Sieron, K., Mahgoub, A.N., 2016, Palaeomagnetic dating of two recent lava flows from Ceboruco volcano, western Mexico: Geophysical Journal International, 207(2), 1203-1215.

Bullard, F.M., 1976, Volcanoes of the Earth: Austin, University of Texas Press, 579 p.

Coe, R.S., 1967, Paleo-intensities of the Earth's magnetic field determined from Tertiary and Quaternary rocks: Journal of Geophysical Research, 72(12), 3247-3262.

Coe, R.S., Grommé, S., Mankinen, E.A., 1978, Geomagnetic paleointensities from radiocarbon-dated lava flows on Hawaii and the question of the Pacific nondipole low: Journal of Geophysical Research, Solid Earth, 83(B4), 1740-1756.

Delgado-Granados, H., Molinero, R., Cervantes, P., Nieto-Obregón, J., Lozano-Santa Cruz, R., Macías-González, H.L., MéndozaRosales, G., Silva-Romo, G., 1998, Geology of Xitle volcano in southern Mexico City -A 
2000-year-old monogenetic volcano in an urban area: Revista Mexicana de Ciencias Geológicas, 15(2), 115-131.

Fergusson, G.J., Libby, W.F., 1963, UCLA radiocarbon dates II: Radiocarbon, 5, 1-22.

Fergusson, G.J., Libby, W.F., 1964, UCLA radiocarbon dates III: Radiocarbon, 6, 318-339.

Fisher, R., 1953, Dispersion on a sphere: Proceedings of the Royal Society of London A, Mathematical and Physical Sciences, 217(1130), 295-305.

García-Quintana, A., Goguitchaichvili, A., Morales, J., Cervantes-Solano, M., Osorio-Ocampo, S., Macías, J.L., UrrutiaFucugauchi, J., 2016, Datación magnética de rocas volcánicas formadas durante el Holoceno: caso de flujos de lava alrededor del Lago de Pátzcuaro (campo volcánico Michoacán-Guanajuato): Revista Mexicana de Ciencias Geológicas, 33(2), 209-220.

González, S., Pastrana, A., Siebe, G., Duller, G., 2000, Timing of the prehistoric eruption of Xitle Volcano and the abandonment of Cuicuilco Pyramid, Southern Basin of Mexico: Geological Society, London, Special Publications, 171, 205-224.

Heizer, R.F., Bennyhoff, J.A., 1958, Archeological investigations of Cuicuilco, Valley of Mexico, 1957: Science, 127(3292), 232-233.

Herrero-Bervera, E., Urrutia-Fucugauchi, J., Martin-del Pozzo, A.L., Böhnel, H., Guerrero, J., 1986, Normal amplitude Brunhes paleosecular variation at lowlatitudes: a paleomagnetic record from the Trans-Mexican Volcanic Belt: Geophysical Research Letters, 13(13), 1442-1445.

Kirschvink, J.L., 1980, The least-squares line and plane and the analysis of palaeomagnetic data: Geophysical Journal International, 62(3), 699-718.

Levi, S., Merrill, R.T., 1978, Properties of single-domain, pseudo-single-domain, and multidomain magnetite: Journal of Geophysical Research, Solid Earth, 83(B1), 309-323.

Luhr, J.F., Carmichael, I.S., 1985, Jorullo Volcano, Michoacán, Mexico (1759-1774): the earliest stages of fractionation in calc-alkaline magmas: Contributions to Mineralogy and Petrology, 90(2-3), 142-161.

Luhr, J.F., Simkin, T., 1993, Parícutin: the volcano born in a Mexican cornfield: Phoenix, Geoscience Press, 427 p.

Marquardt, D.W., 1963, An algorithm for leastsquares estimation of non-linear parameters: Journal of the Society for Industrial and Applied Mathematics(2), 11, 431-441.

Mahgoub, A.N., Böhnel, H., Siebe, C., Chevrel, M.O., 2017, Paleomagnetic study of El Metate shield volcano (Michoacán, Mexico) confirms its monogenetic nature and young age ( $1250 \mathrm{CE})$ : Journal of Volcanology and Geothermal Research, 336, 209-218.

Martin Del Pozzo, A.L., 1982, Monogenetic vulcanism in sierra Chichinautzin, Mexico: Bulletin Volcanologique, 45(1), 9-24.

Martin-Del Pozzo, A.L., Cordoba, C., López, J., 1997, Volcanic impact on the southern Basin of Mexico during the Holocene: Quaternary International, 43-44, 181-190.

Muller, F., 1990, La cerámica de Cuicuilco B. Un rescate arqueológico: México, Colección Científica, Instituto Nacional de Antropología e Historia, 186, 285 p.

Navarrete, C., 1991, Cuicuilco y la arqueología del Pedregal: Crónica de un desperdicio: Arqueología, 5, 69-84.

Pavón-Carrasco, F.J., Rodríguez-Gonzáles, J., Osete, M.L., Torta, J.M., 2011, A Matlab tool for archaeomagnetic dating: Journal of Archaeological Science, 32(8), 408-419.

Pavón-Carrasco, FJ., Osete, M.L., Torta, J.M., De Santis, A., 2014, A geomagnetic field model for the Holocene based on archaeomagnetic and lava flow data: Earth and Planetary Science Letters, 388, 98-109. 
Pérez-Rodríguez, N., Morales-Contreras, J., García-Tenorio, F., Goguitchaichvili, A., 2015, Fechamiento arqueomagnético de las últimas tres erupciones plinianas del volcán Popocatépetl, en Rapalini, A.E., Caballero-Miranda, C., Goguitchaichvili, A. (eds.), Proceedings of the Fourth Biennal of Latinmag: São Paulo, Brasil, Latinmag Letters, Special Issue, 6(C11), 1-7.

Piña-Chan, R., 1967, Un complejo Coyotlatelco en Coyoacán, México, D.F.: Anales de Antropología, 4(1), 141-160.

Siebe, C., 2000, Age and archaeological implications of Xitle volcano, southwestern Basin of Mexico-City: Journal of Volcanology and Geothermal Research, 104(1-4), 45-64.

Speranza, F., Branca, S., Coltelli, M., D’Ajello Caracciolo, F., Vigliotti, L., 2006, How accurate is "paleomagnetic dating"? New evidence from historical lavas from Mount Etna: Journal of Geophysical Research, Solid Earth, 111(B12), B12S33.

Thellier, E., Thellier, O., 1959, Sur l'intensité du champ magnétique terrestre dans le passé historique et géologique: Annales de Géophysique, 15(3), 285-376.
Urrutia-Fucugauchi, J., 1996, Paleomagnetic study of the Xitle-Pedregal de San Angel lava flow, southern basin of Mexico: Physics of the Earth and Planetary Interiors, 97(1), 177-196.

Urrutia-Fucugauchi, J., Martin-del Pozzo, A.L., 1993, Implicaciones de los datos paleomagnéticos sobre la edad de la Sierra de Chichinautzin, Guenca de México: Geofísica Internacional, 32(3), 523-533.

Urrutia-Fucugauchi, J., Goguitchaichvili, A., Pérez-Cruz, L., Morales, J., 2016, Archaeomagnetic dating of the eruption of Xitle Volcano, Basin of Mexico: Implications for the mesoamerican centers of Cuicuilco and Teotihuacan: Arqueología Iberoamericana, 30, 23-29.

Webring, M., 1985, SAKI: a FORTRAN program for generalized linear inversion of gravity and magnetic profiles: Virginia, United States Geological Survey, Open file Report, 85-122, 108 p.

Zijderveld, J.D.A., 1967, AC demagnetization of rocks: analysis of results, en Collinson, D., Creer, K.M., Runcorn, S.K. (eds.), Methods in paleomagnetism, 3, 254-286. 